\title{
Low-frequency complex magnetic susceptibility of magnetic composite microspheres in colloidal dispersion
}

\author{
Ben H. Erné*, Maria Claesson, Stefano Sacanna, Mark Klokkenburg, \\ Emile Bakelaar, Bonny W.M. Kuipers
}

Van 't Hoff Laboratory for Physical and Colloid Chemistry, Chemistry Department, Science Faculty, Utrecht University, The Netherlands

Available online 8 December 2006

\begin{abstract}
A procedure is presented to determine the permanent magnetic dipole moment of composite microspheres containing magnetic nanoparticles with a blocked magnetic dipole moment. The composite particles are dispersed in a solvent, and the complex magnetic susceptibility is measured from 0.1 to $1000 \mathrm{~Hz}$ using a highly sensitive new setup. Composite particles with a permanent magnetic dipole moment are revealed by a characteristic frequency that corresponds to the Brownian rotation of the microspheres. From measured susceptibility spectra, we calculate the permanent magnetic dipole moment of recently developed cobalt ferrite-doped silica and latex microspheres.
\end{abstract}

(C) 2006 Elsevier B.V. All rights reserved.

Keywords: Complex magnetic susceptibility; Brownian relaxation; Magnetic dipole moment; Microsphere; Magnetic nanoparticle; Colloidal dispersion; Cobalt ferrite; Silica; Latex; Magnetic susceptibility

Composite microspheres with embedded nanoparticles whose magnetic moment is blocked are likely to have a permanent magnetic dipole moment in zero field. This depends on the number of nanoparticle dipoles and their orientations, a function of the magnetic field treatment history of the microparticles. In biomedical applications, the surface of magnetic microspheres can, for instance, be covered with bioreceptors so that targeted molecules can be isolated from a serum using a magnet [1], but the microspheres generally do not have a permanent magnetic dipole moment in zero field; they acquire an induced magnetic moment in an external magnetic field. New composite microspheres from our laboratory incorporate nanoparticles whose magnetic moment is blocked at room temperature $[2,3]$ to make them suitable for the fundamental study of the rotational diffusion of spheres at very high colloid concentrations, which are difficult to study by optical techniques. The permanent magnetic moment of such composite particles cannot be determined in the same way as for single-domain nanoparticles. We present an

\footnotetext{
${ }^{*}$ Corresponding author. Tel.: + 31302532934 ; fax: + 31302533870 .

E-mail address: b.erne@chem.uu.nl (B.H. Erné).
}

approach based on measuring the complex magnetic susceptibility of a colloidal dispersion at frequencies in the range of the Brownian rotation rate of the composite particles.

To measure the permanent magnetic dipole moment of composite microspheres, the first step is to make a stable colloidal dispersion of the particles in a solvent. The wrong way would be to measure the magnetic response of dry composite particles, since that would correspond to the behavior of immobile nanoparticles, independent of the size of the composite microspheres in which they are embedded. A magnetic study of dry composite microspheres would therefore not give information about the permanent magnetic moment of the microspheres. This situation changes when the composite microspheres are free to rotate in a solvent. Now, the magnetization of the colloidal dispersion of composite particles in a weak external magnetic field depends on the permanent magnetic dipole moment of the composite particles according to the Langevin equation for paramagnetic systems [4]:

$$
M / M_{\mathrm{s}}^{*}=\operatorname{coth}\left[\mu_{0} \mu_{\text {micro }}^{*} H /\left(k_{\mathrm{B}} T\right)\right]-\left[k_{\mathrm{B}} T /\left(\mu_{0} \mu_{\mathrm{micro}}^{*} H\right)\right]
$$


under the condition that

$\left[\mu_{0} \mu_{\text {nano }} H /\left(k_{\mathrm{B}} T\right)\right] \ll 1$,

where $M$ is the magnetization of the dispersion, $M_{\mathrm{S}}^{*}$ is the hypothetical saturation magnetization upon full alignment of composite particles whose magnetic dipole moment would be the same as in zero field, $\mu_{0}$ is the permeability of vacuum, $\mu_{\text {micro }}^{*}$ is the zero-field permanent magnetic dipole moment of the composite microparticles, $H$ is the magnetic field (in $\mathrm{A} / \mathrm{m}$ ), $k_{\mathrm{B}}$ is the Boltzmann constant, $T$ is the absolute temperature, and $\mu_{\text {nano }}$ is the magnetic dipole moment of the embedded nanoparticles. Eq. (1a) describes the competition between alignment of the composite particles in the magnetic field and loss of alignment by thermal motion. The complication is that before the magnetic field is sufficiently high to align the dipole moments of the composite particles and a magnetization $M_{\mathrm{s}}^{*}$ is reached, the dipoles of individual nanoparticles also start to be aligned inside the microspheres. As a result, the dipole moment of the microspheres is no longer the same as in zero field. The magnetization does not reach a plateau at $M_{\mathrm{s}}^{*}$ but it increases until all nanoparticle dipoles are aligned, whether they are embedded in microparticles or not. Information about the permanent magnetic moment of the composite particles can therefore best be obtained from measurements using weak magnetic fields that fulfill the condition stated in Eq. (1b): weak fields that are sufficient to cause partial alignment of the microspheres but that have a negligible interaction with individual nanoparticles.

Determination of the microsphere permanent dipole moment requires magnetic susceptibility measurements as a function of frequency. In the low-field and low-frequency limits, the dimensionless magnetic susceptibility $\chi=M / H$ of a colloidal dispersion consists of two contributions, $\chi=\chi_{\mathrm{HF}}+\chi_{\mathrm{LF}}$. One contribution, $\chi_{\mathrm{LF}}$, exists only at frequencies that are sufficiently low that the orientation of the permanent dipole moment of microspheres can respond to a weak alternating external magnetic field by Brownian rotation:

$\chi_{\mathrm{LF}}=\left(N_{\text {micro }} / V\right) \mu_{0}\left(\mu_{\text {micro }}^{*}\right)^{2} /\left(3 k_{\mathrm{B}} T\right)$,

where $N_{\text {micro }}$ is the number of composite microparticles and $V$ is the sample volume. Eq. (2) is obtained by Taylor expansion of Eq. (1a). From Eq. (2), measurement of the initial susceptibility of a sample with a known concentration $N_{\text {micro }} / V$ allows calculation of the permanent magnetic moment $\mu_{\text {micro }}^{*}$ of the composites. The second contribution, $\chi_{\mathrm{HF}}$, also exists at higher frequencies where the contribution $\chi_{\mathrm{LF}}$ of the microspheres has been lost. It is due to nanoparticles that respond individually, that is, nanoparticles that exhibit Néel relaxation or that are not embedded in microparticles. It is to separate the contribution $\chi_{\mathrm{LF}}$ of the permanent microparticle dipoles from the other contributions $\chi_{\mathrm{HF}}$ that $\chi$ is measured as a function of frequency.
The characteristic frequency of a colloidal dispersion of particles with a permanent magnetic moment is determined by the rate of Brownian rotation of the particles in the solvent $[6,7]$ :

$\omega_{\mathrm{B}}=k_{\mathrm{B}} T /\left(4 \pi \eta a_{\mathrm{H}}^{3}\right)$,

where $\eta$ is the viscosity of the solvent and $a_{\mathrm{H}}$ is the hydrodynamic radius of the particles, assumed to be spherical. At this frequency, the in-phase, real component $\chi^{\prime}$ of the complex susceptibility has the highest frequency dependence and the out-of-phase, imaginary component $\chi^{\prime \prime}$ has an extremum $[5,6]$ :

$\chi=\chi^{\prime}-\mathrm{i} \chi^{\prime \prime}$,

$\chi^{\prime}=\chi_{0} \omega_{\mathrm{B}}^{2} /\left(\omega_{\mathrm{B}}^{2}+\omega^{2}\right)$,

$\chi^{\prime \prime}=\chi_{0} \omega_{\mathrm{B}} \omega /\left(\omega_{\mathrm{B}}^{2}+\omega^{2}\right)$,

where $\omega=2 \pi f$ is the angular frequency in $\mathrm{s}^{-1}$ and $f$ is the cycle frequency in $\mathrm{Hz}$. If a switch from the low-frequency limit value to zero occurs around the frequency corresponding to the hydrodynamic radius of the composite particles, it can be concluded that the composite particles have a permanent magnetic moment and that all the hardmagnetic particles present are embedded in composite particles. A composite particle without a permanent magnetic moment, for instance, based on nanoparticles that relax by Néel rotation inside the crystals [6,7], would yield a frequency-independent real susceptibility up to much higher frequencies than those corresponding to Brownian rotation of the particles. Nanoparticles with a blocked magnetic moment and that are not embedded inside composite particles would give a relaxation rate around the frequency corresponding to the rotation of nanoparticles and would therefore be easily detected: from Eq. (3) for a viscosity of $\eta \approx 1 \mathrm{mPas}$ at $298 \mathrm{~K}$, the characteristic frequencies for the Brownian rotation of composite particles of $100-1000 \mathrm{~nm}$ in radius is in the $0.05-50 \mathrm{~Hz}$ range and that of nanoparticles of $1-10 \mathrm{~nm}$ in radius is in the $0.05-50 \mathrm{MHz}$ range, 3-6 orders of magnitude higher.

Complex magnetic susceptibility measurements were performed using a setup whose details will be presented elsewhere [8], a much higher sensitivity and lower frequency version of a setup that was presented earlier [7]. In brief, the setup is a differential transformer with two multilayered cylindrical copper coils, with primary layers through which an applied alternating current flows and secondary layers in which an alternating voltage is induced. The key new idea of the setup is to use secondary coils with a maximized number of layers beyond which the signal would no longer increase but drop due to capacitance and absorption effects. An AC current is applied to the primary coils using a Yokogawa FG120 function generator and the voltage induced in the secondary coils is measured using a 
7265 Perkin-Elmer differential lock-in amplifier in the $2 \mathrm{mHz}$ to $250 \mathrm{kHz}$ range. The colloidal dispersion of magnetic microspheres is contained in a glass tube. When the magnetic sample is introduced in the coils, the change in the measured alternating voltage is proportional to the complex susceptibility of the sample. Measurements above $1 \mathrm{kHz}$ are dominated by artifacts due to multiple resonances in the electrical impedance of the coils and due to absorption of the magnetic field by the copper wires, but below $1 \mathrm{kHz}$, susceptibilities can be measured with a high sensitivity, sufficient to determine the diamagnetic susceptibility of simple solvents around $10 \mathrm{~Hz}$. The setup was calibrated using different ferrofluids and calibration standards. The amplitude of the alternating magnetic field was kept very low, at most about $50 \mathrm{~A} / \mathrm{m}$, on the order of the earth magnetic field, to avoid changing the magnetic structure of the samples.

The chemical synthesis of the investigated composite magnetic particles is presented in detail elsewhere. The magnetic silica particles were obtained by covering silica spheres with a layer of cobalt ferrite nanoparticles and adding an outer layer of silica with a coating of 3(trimethoxylsilyl)propyl metacrylate (TPM) [2]. The magnetic latex spheres were obtained by first forming a monodisperse Pickering emulsion with cobalt ferrite nanoparticles ( $13 \mathrm{~nm}$ in average radius) and a polymerizable silane-coupling agent [3], and then incorporating the resulting droplets into a PMMA shell via a standard emulsion polymerization [9]. The concentrations of the dispersions were determined by weighing a known volume of dispersion after evaporation of the solvent. Particle diameters were obtained by electron microscopy and angledependent measurements of static and dynamic light scattering using homebuilt setups [9]. Magnetization curves of the colloidal dispersions were measured using a Micromag 2900 alternating gradient magnetometer (Princeton Measurements Corp.).

Fig. 1a and b shows complex magnetic susceptibility spectra of dispersions of silica and latex composite microspheres with embedded hard-magnetic nanoparticles. Note the low-frequency range, starting at $0.1 \mathrm{~Hz}$, and the relatively low susceptibilities. Until now, complex susceptibility measurements on magnetic dispersions have mainly been presented for concentrated ferrofluids, with spectra starting at $100 \mathrm{~Hz}$ or higher and susceptibilities $\chi>1$. For comparison, a dispersion of hard-magnetic iron nanoparticles with a hydrodynamic radius of $13 \mathrm{~nm}$ has a much higher characteristic frequency for Brownian rotation, about $4000 \mathrm{~Hz}$ [7]. In an aqueous dispersion of $1 \mu \mathrm{m}$ Dynabeads (MyOne carboxylic acid), no relaxation is observed at $0.5 \mathrm{~Hz}$, which is the expected characteristic frequency for Brownian rotation (Eq. (3)), because the magnetic moment relaxes inside the soft-magnetic microspheres [10].

The hydrodynamic radius of the microspheres can be calculated from the characteristic frequency for Brownian rotation. The curves through the measured points in Fig. 1

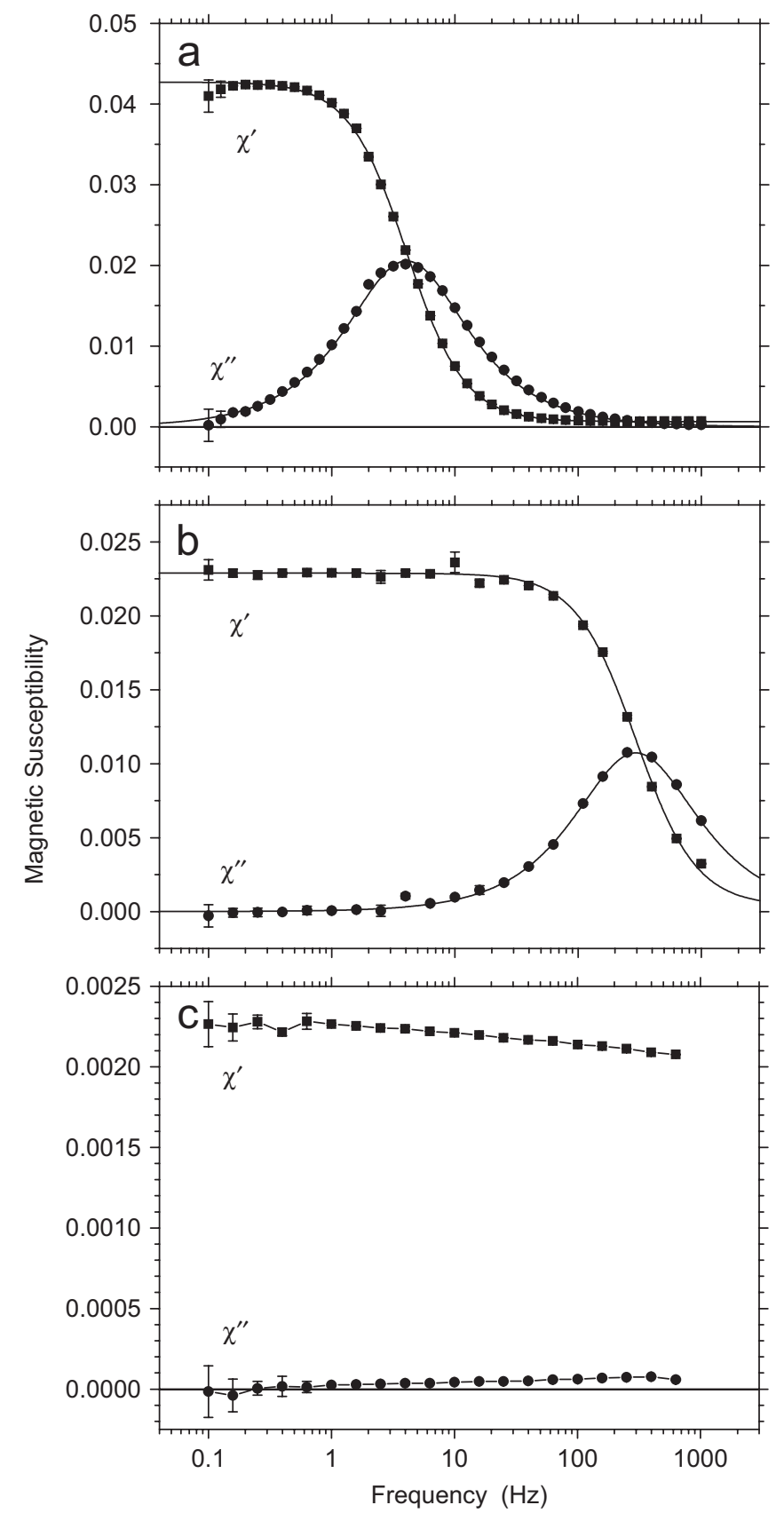

Fig. 1. Complex magnetic susceptibility spectra at $296 \mathrm{~K}$ of: (a) spherical magnetic silica particles in ethanol $\left(a_{\mathrm{H}} \approx 228 \mathrm{~nm}\right)$, (b) spherical magnetic latex particles in water $\left(a_{\mathrm{H}} \approx 58 \mathrm{~nm}\right)$, and (c) Dynabeads $\left(a_{\mathrm{H}} \approx 500 \mathrm{~nm}\right)$. The particles in (a) and (b) have a permanent magnetic dipole moment because of embedded cobalt ferrite nanoparticles $(r \approx 8 \mathrm{~nm})$. If the Dynabeads (c) had a permanent dipole moment, the spectrum would show a Brownian relaxation at $0.5 \mathrm{~Hz}$ (Eq. (3) with $\eta=0.89 \mathrm{mPas}$ and $T=298 \mathrm{~K})$.

are least-squares fits of the spectra on the basis of Eqs. (3)-(4) and a log-normal distribution of the hydrodynamic radius $a_{\mathrm{H}}[11]$ around a value of $\left\langle a_{\mathrm{H}}\right\rangle$ with a variance $\sigma^{2}$. From the characteristic frequencies $\left(\left\langle a_{\mathrm{H}}\right\rangle=4.05 \pm 0.03 \mathrm{~Hz}\right.$ and $\sigma=0.225 \pm 0.005$ for silica and $\left\langle a_{\mathrm{H}}\right\rangle=294.9 \pm 1.4 \mathrm{~Hz}$ and $\sigma=0.32 \pm 0.01$ for latex), hydrodynamic radii of $228 \pm 17 \mathrm{~nm}$ (silica) and $58 \pm 6 \mathrm{~nm}$ 
(latex) are obtained. The fact that the susceptibility drops to zero not far above the characteristic frequency for Brownian rotation of the microparticle indicates that all the magnetic nanoparticles present are embedded in composite particles and that they have blocked orientations of the magnetic dipole moment.

The hydrodynamic radii from complex magnetic susceptibility measurements can be compared to determinations using other techniques. The hydrodynamic radius is expected to be slightly larger than the physical radius. Effectively, a shell of solvent rotates along with the particles, for instance, because of Van der Waals attraction and hydrodynamic interactions that are a function of surface roughness. The hydrodynamic radius of the silica particles is of the order expected from electron microscopy, which gives a physical radius of $195 \pm 10 \mathrm{~nm}$ (Fig. 2a). It is remarked that the slightly porous silica microparticles tend to shrink under electron microscopy conditions, so that electron microscopy underestimates the physical radius of

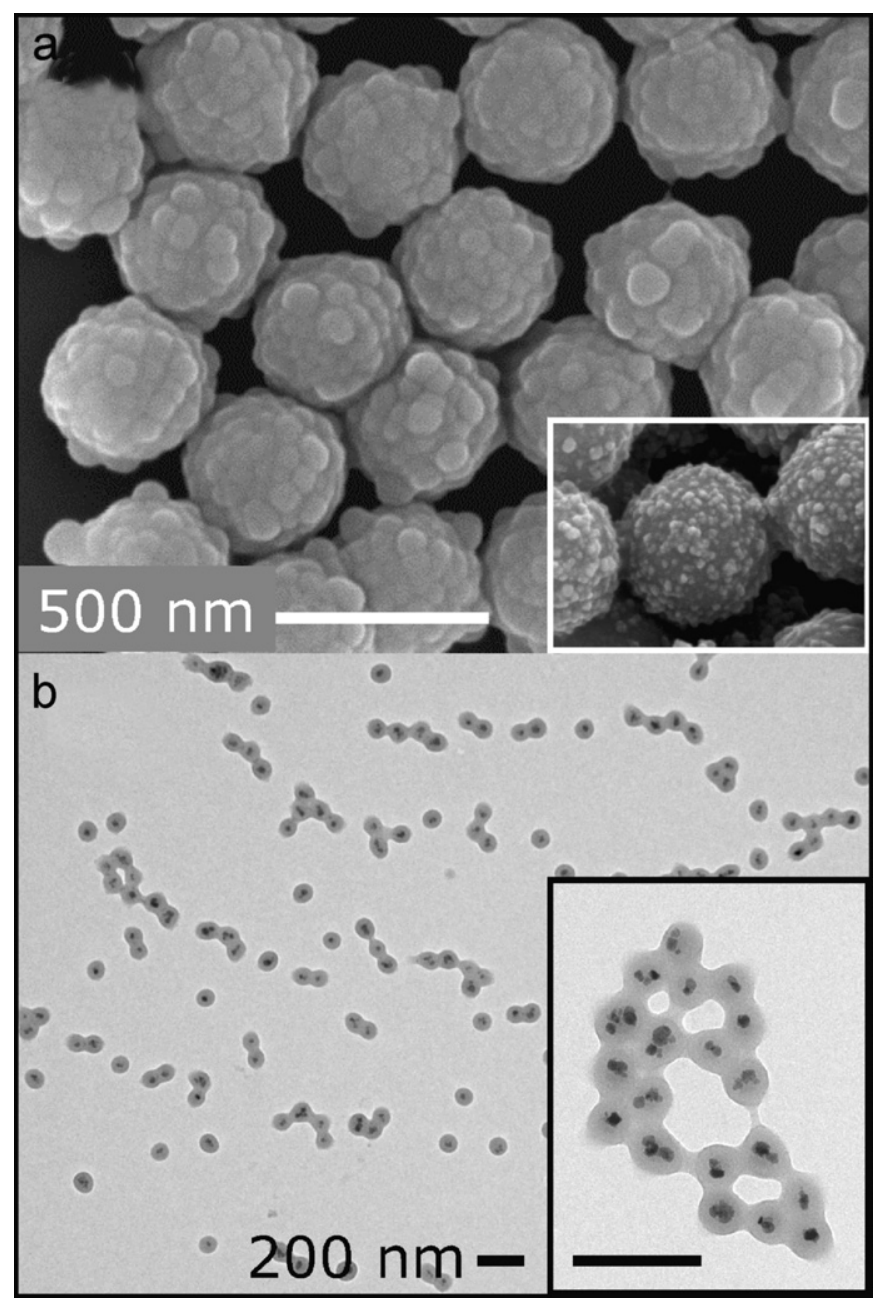

Fig. 2. Electron microscopy images of the particles of Fig. 1. (a) Spherical magnetic silica particles; the inset shows the particles without their outer silica layer, so that the shell of nanoparticles becomes visible and (b) spherical magnetic latex particles. Due to melting in the electron beam, the latex particles lose their spherical shape. The black dots are the embedded nanoparticles. the particles in the colloidal dispersion. The size of the latex particles is more difficult to determine from other techniques: in the electron microscope, the latex particles melt (Fig. 2b), and although static light scattering gives a Guinier radius of about $50 \mathrm{~nm}$, this is a rough estimate because the particles are not optically homogeneous. Dynamic light scattering gives the same hydrodynamic radius as found here from the complex magnetic susceptibility spectrum, namely $58 \mathrm{~nm}$. The low polydispersity of the hydrodynamic radii agrees with the observation of colloidal crystal formation in sediments of the particles.

The zero-field magnetic moment of the microparticles can be calculated using Eq. (2) and requires knowing the colloidal concentration $N_{\text {micro }} / V$ and the contribution $\chi$ of the microspheres to the low-frequency limit of the magnetic susceptibility. In Fig. 1, the silica particle concentration is $7.84 \times 10^{17} \mathrm{~m}^{-3}$ and the latex particle concentration is $1.2 \times 10^{19} \mathrm{~m}^{-3}$. The fitted low- and high-frequency limits for the magnetic silica dispersion are $\chi=0.0427 \pm 0.0004$ and $0.00062 \pm 0.00002$ and for the latex dispersion they are $\chi=0.0228 \pm 0.0004$ and $\chi=0.00038 \pm 0.00010$. This corresponds to permanent magnetic dipole moments of $\mu_{\text {micro }} \approx 2.3 \times 10^{-17} \mathrm{~A} \mathrm{~m}^{2}$ for the magnetic silica and $\mu_{\text {micro }} \approx 4.2 \times 10^{-18} \mathrm{~A} \mathrm{~m}^{2}$ for the magnetic latex. As expected, these values are higher than the magnetic moment of a single nanoparticle $\left(\mu_{\text {nano }} \approx 1.0 \times 10^{-18} \mathrm{~A} \mathrm{~m}^{2}\right)$ but lower than the magnetic moment of the microparticle in a saturating magnetic field $\left(1.2 \times 10^{-15} \mathrm{~A} \mathrm{~m}^{2}\right.$ for the magnetic silica particles and $6 \times 10^{-18} \mathrm{~A} \mathrm{~m}^{2}$ for the magnetic latex particles).

It can now be verified that the measurements were performed in the weak-field limit, for the nanoparticle dipoles as well as for the microparticle dipoles, leaving the magnetic structure of the samples unchanged by the weak alternating magnetic field. The Langevin parameter $\left[\mu_{0} \mu_{\text {micro }}^{*} H /\left(k_{\mathrm{B}} T\right)\right]$ (Eq. (1a)) was about 0.35 for the silica microparticles during the complex susceptibility measurements $(H=50 \mathrm{~A} / \mathrm{m})$. This interaction is weak but not completely negligible, and the low-frequency limit was found to increase a few percent upon decreasing the field amplitude; this issue will be addressed in detail by us in future work. For the latex particles and the single nanoparticles, the Langevin parameter remained lower, below 0.1. Below a Langevin parameter of 1 , no new structures are expected due to the external magnetic field $[12,13]$. Zero-field dipolar structures are not expected either, since the maximum magnetic interaction between two composite particles, as estimated by $\mu_{0}\left(\mu_{\text {micro }}^{*}\right)^{2} /\left(16 \pi a_{\mathrm{H}}^{3}\right)$, remained below $0.3 k_{\mathrm{B}} T$, for the magnetic silica particles and below $0.6 k_{\mathrm{B}} T$ for the magnetic latex particles, whereas zero-field dipolar structures are observed when the head-to-tail dipolar pair interaction energy is clearly more than $2 k_{\mathrm{B}} T$ [14-16] . The expression $\mu_{0}\left(\mu_{\text {micro }}^{*}\right)^{2} /\left(16 \pi a_{\mathrm{H}}^{3}\right)$ assumes point dipoles and therefore only gives a rough estimate of the interaction between composite microparticles. In the latex particles, the magnetic material is indeed at the core, whereas in the 
silica particles, the magnetic material is in a shell near the surface of the spheres.

In line with the weakness of the dipolar interactions between the microspheres in zero field, no aggregates of microspheres are detected in the complex magnetic susceptibility spectra. Clusters of microspheres would lead to several times lower characteristic frequencies for Brownian rotation than for single microspheres [7]. The possibility to detect aggregation or to assess the probability of aggregation due to magnetic forces is an important feature of the technique. Colloidal stability is a prerequisite in most applications of magnetic microspheres.

Although insufficient for aggregation, interactions of $1 k_{\mathrm{B}} T$ or less between microparticles might still affect the Brownian rotation in a detectable way. Adsorption of molecules at the surface of the microparticles would also decrease the rotation rate, by increasing the hydrodynamic radius of the particles. The latter effect can be used to develop highly sensitive biomolecular sensing procedures, as was demonstrated by Astalan et al. [17]. The interaction of the microspheres with even larger objects could drastically slow down the motion of the microspheres. In the near future, we will study the rotational dynamics of our microspheres with a permanent magnetic moment in confined geometries such as colloidal crystals or glasses. In such studies, magnetic detection of the Brownian rotation rate has definite advantages over optical techniques used in the past. Their interpretation is complicated by multiple optical scattering effects and their use puts difficult requirements on the optical properties of the particles [18-20].

In conclusion, the permanent magnetic dipole moment of composite microparticles with embedded nanoparticles must be determined by measurements on colloidal dispersions of the microparticles in the weak magnetic field limit. From the concentration of the dispersion and the initial magnetic susceptibility, the permanent magnetic moment can be calculated. The frequency dependence of the complex magnetic susceptibility provides information about the rotational diffusion rate of the particles and the presence or absence of magnetic nanoparticles that are not embedded in the composites. From the rotational diffusion rate, the hydrodynamic radius of the particles can be calculated. Interactions of the particles with each other or with adsorbants are likely to lower the rotational diffusion rate. On that basis, the particles could be used as a magnetic probe of the chemical environment in which they are dispersed.

\section{References}

[1] Q.A. Pankhurst, J. Connolly, S.K. Jones, J. Dobson, J. Phys. D 36 (2003) R167.

[2] E.M. Claesson, A.P. Philipse, Langmuir 21 (2005) 9412.

[3] S. Sacanna, A.P. Philipse, Langmuir 22 (2006) 10209.

[4] D. Jiles, Introduction to Magnetism and Magnetic Materials, Chapman \& Hall, New York, 1991.

[5] R.E. Rosensweig, J. Magn. Magn. Mater. 252 (2002) 370.

[6] P.C. Fannin, Adv. Chem. Phys. 104 (1998) 181.

[7] B.H. Erné, K. Butter, B.W.M. Kuipers, G.J. Vroege, Langmuir 19 (2003) 8218.

[8] B.W.M. Kuipers, I.A. Bakelaar, M. Klokkenburg, B.H. Erné, submitted.

[9] G.H. Koenderink, S. Sacanna, et al., Langmuir 17 (2001) 6086.

[10] G. Fonnum, C. Johansson, et al., J. Magn. Magn. Mater. 293 (2005) 41.

[11] B.H. Erné, E. van den Pol, G.J. Vroege, et al., Langmuir 21 (2005) 1802.

[12] Y. Lalatonne, J. Richardi, M.P. Pileni, Nat. Mater. 3 (2004) 121.

[13] C. Holm, J.-J. Weis, Curr. Opin. Colloid Interface Sci. 10 (2005) 133.

[14] K. Butter, P.H. Bomans, P.M. Frederik, et al., Nat. Mater. 2 (2003) 88.

[15] M. Klokkenburg, C. Vonk, et al., J. Am. Chem. Soc. 126 (2004) 16706.

[16] M. Klokkenburg, R.P.A. Dullens, et al., Phys. Rev. Lett. 96 (2006) 037203.

[17] A.P. Astalan, F. Ahrentorp, C. Johansson, et al., Biosens. Bioelec. 19 (2004) 945.

[18] G.H. Koenderink, S. Sacanna, et al., Phys. Rev. E 69 (2004) 021804.

[19] M.P. Lettinga, G.H. Koenderink, et al., J. Chem. Phys. 120 (2004) 4517.

[20] G.H. Koenderink, M.P. Lettinga, A.P. Philipse, J. Chem. Phys. 117 (2002) 7751. 